\title{
Schweizerische Ophthalmologische Gesellschaft
}

44. Generalversammlung in Glarus, 28.-30. September 1951

Société Suisse d'Ophtalmologie

44e Assemblée generate à Claris, 28-30 septembre 1951

Verzeichnis der Vorträge - Liste des communications

Thíel, R.: Zur Pathogenese des primäre $\pi$ Glaukoms . . 19õ

Diskussion: Rintelen, Goldmann. Schlußwort: Thiel. Huber, O.: Ophthalmologisches aus dem

Glarnerland . 198 Goldmann, H.: Die Wirkungsweise der Iridektomie beim

chronisch kongestiven Glaukom 202

Hoffmann-Egg, L.: Sympathische Ophthalmie nach Iriden-

cleisis mit ausgedehnter epiretinaler Zellanhäufung liber

den Netzhautgefäßen 207

Diskussion: Moeschlin.

Böhringer, H.R.: Sekundärglaukom mit Gefäßneubildungen

auf der Iris 211

Zollinger, R.: Klinische Untersuchungen über Gefäßneubildungen auf der Iris

Diskussion: Goldmann, Rintelen. Schlußwort: Zollinger.

Franceschetti, A.: Le strabisme concomitant aigu . . . 219

Discussion: Dufour, Amsler. Hegner, H. J.: Zur Therapie der Augenmuskelverletzungen.

(Manuskript nicht eingegangen) 227

Diskussion: Rintelen, Franceschetti, Meyer. Rintelen, F.: Zur Frage der Blutungsprophylaxe bei bulbus-

eröffnenden Operationen $\quad, 228$

Diskussion: Thiel, Huber, Amsler, Dufour, R. Rrückner, Böhringer. Schlußwort: Rintelen.

Streiff, E. B., et E. Rosselet: Plaies perforantes, foyers in-

fectieux, ophtalmie sympathique 235

Discussion: Verrey, Dufour.

Bangerter, A.: Neue kleine Plastiken (mil Projektion). (Manuskript nicht eingegangen)

Diskussion: Rintelen.

Amsler, M.: Kystes de Гiris 241

Discussion: Goldmann.

Bürkí, E.: Über den klinischen Wert der Röntgenaufnahme

des Canalis opticus 243

Diskussion: Thiel, Huber, A. Bruckner, Rintelen. Schlußwort: Bürki.

Dufour, R., et /. F. Cuendet: Appreciation quantitative desperturbations des functions visuelles.

(A propos d'un casde traumatisme du nerf optique) 249

Franceschetti, A., et /. H. Rickli: Névrite rétrobulbaire après

intoxication aiguë aux hydrocarbures chlorés . . 255

Huber, A.: Einseitige Stauungspapille, Pseudoneuritis und

Pseudopapillenödem 261 
Diskussion: A. Bruckner, Goldmann.

$17^{*}$

Amsler, M., und L. Hoffmann-Egg: Eine lochverdächtige

Stelle in der Netzhaut 269

Diskussion: Goldmann.

V err eg, F.: Kritische Studien über Mißerfolge bei Netzhaut-

operationen. (1. Mitteilung) 271

Diskussion: A. Bruckner. Schlußwort: V"errey.

Goldmann, H.: Em neues Spaltlampenfluorometer . . 277

Diskussion: Huber. Schlußwort: Goldmann.

Witmer, R.: Elektrophorese des Kammerwassers . . . 280

Diskussion: Amsler, Goldmann, Verrey. Blum, J. D.: Aphakie unilatérale et verres de contact . .

284 Dufour, R.: Indications particulières des verres de contact :

kératite neuroparalytique, albinisme 290

Discussion à Blum et Dufour: Amsler, Dufour, Goldmann, Verrey. Conclusion: Dufour.

Portmann, U. P., et M. Dufour: Indications et contre-indi-cations données par Torthoptique dans le traitement du

strabisme convergent concomitant 294

Discussion: Rintelen, Meyer, Kretzschmar. Conclusion: Portmann.

Saubemuinn, G.: Die Bedeutung des Applikationsmodvis bei

antibiotischer Therapie 303

Diskussion: Dufour. Schlußwort: Saubermann.

Müller, P.: Cortison. Indikation und Anwendung . . . 310Moser, R.: Gefahren der

Cortisontherapie $\quad 313$

Diskussion: Franceschetti, Goldmann, Saubermann.

Neuenschwande $\Gamma$, M.: Homöopathie in der Augenheilkunde 316 Rintelen, F.: Die

Farbenphotographie im klinischen Unter-

richt 320

Schlußwort: Rintelen.

Bischler, V.: Le Pseudo-Phénomène de Graefe congenital . 322 Messikommer, W.: Akuter

Keratokonus bei einem Kinde . 326

Diskussion: Rintelen, Böck. Witmer, R.: Erfahrungen mit dem Sero-Test auf Tuberku-

lose nach Middlebrook und Dubos 329

Diskussion: Böck.

Cuendet, J.-F., E. Rosselet et C. Meneghini: Observations cliniques avec des nouveaux collyres isotoniques et tam-

ponnés 334

Barth, J.: Dystrophia myotonica 338

Wettler, H.: Über Augensymptome bei Gargoylismus . . 338 Kretzschmar, S.: A propos de la fausse correspondance ré-

tinienne $\quad 343$

Discussion: Goldmann.

- La coordimétrie au périmètre de Goldmann . . . 348

Witmer, R.: Uveitis chronica bei Bronchiektasien . . 352 Diskussion: Gruber, Witmer.

- $\quad$ Eine spezielle Form rezidivierender Chorioiditis. (Hier-

zu Tafel I) $\quad 353$ 
W. Weidmann

W.: Vorführung eines neuen Adaptometers . 355

Diskussion: Goldmann. 